\title{
Studien über den intermediären Eiweiss= und Kohlehydrat = stoffwechsel in der Lunge unter verschiedenen Bedingungen.
}

\author{
II. Mitteilung : Bestimmungen bei eingeschränkter \\ Lungenzirkulation und Atemfläche.
}

Von

Sin-itiro Endo.

(透雚潐一郎)

(Aus der Medizinischen Klinik von Prof. Dr. T. Kato, Tohoku Reichsuniversität zu Sendai.)

In der ersten Mitteilung') der einschlägigen Studien ist der Einfluss der Lunge auf die Eiweisskörper und Kohlehydrate des Blutes analysiert worden; es ergab sich, dass beim Durchgang des Blutes durch die normalen Lungen dessen Eiweiss abnimmt, der kolloid-osmotische Druck des Blutes (im folgenden kurz k.o.D.) sich erniedrigt; des weiteren mit Riicksicht auf Kohlehydrate ist festgestellt worden, dass die Zunahme des Blutzuckers und die Abnahme der Blutmilchsäure statthaben. Dabei wurde darauf aufmerksam gemacht, dass die Abnahme des Bluteiweisses und die Erniedrigung des k.o.D. vorwiegend auf die Spaltung kleinförmiger Eiweissteilchen in den Lungen zurückzuführen ist, und ferner dass obige Veränderungen des Blutzuckers und der Blutmilchsäure in der Hauptsache der Funktionsfähigkeit der Lungenzellen zur Zuckerbildung sowie zurMilchsäureelimination zuschreiben müssen. Aus Ergebnissen der weiter daraufhin gerichteten, unter Inhalation von $\mathrm{O}_{2}$-armer bzw. $\mathrm{CO}_{2}$-reicher Luft ausgeführten Untersuchungen wurde der Schluss gezogen, dass die Funktion der Lungen zu oben geschilderter Eiweiss- und Kohlehydratbewältigung erst unter hirreichendem $\mathrm{O}_{2}$-Angebot vollkommen gesteuert wird. Im Zusammenhang mit oben erwähnter Schlussfolgerung erhebt sich erneut die Frage: Können in Fällen, wo aus irgendwelchen Gründen unter anderem namentlich Störungen der Zirkulation der Lunge oder haupt-

1) End o, Tohoka Journ. Exp. Med, 1941, 40, 542: 
sächlich die Einschränkung der Atemfläche der Lunge sich finden, infolge von unzulänglicher Blutversorgung oder mangelhafter $\mathrm{O}_{3}$-Aufnahmefähigkeit die Funktionsänderungen der Lungenzellen, welche zu abnormen Verhalten des Eiweiss- und Kohlehydratstoffwechsels in der Lunge führen, zustande kommen? Um mir Aufschlüsse über diese Frage zu verschaffen, sind einschlägige Studien unter diesem Gesichtspunkt weiter fortgesetzt worden.

Behufs Verkleinerung der Lungenzirkulation oder zwecks Einengung der atmenden Fläche der Lunge sind bei Kaninchen der einseitige Pneumothorax angelegt oder die Pleuritis bzw. Pneumonie experimentell erzeugt worden, es wurde ferner eine Luftkanüle über die Bifurkationsstelle der Trachea hinweg, bis tief in den einen Bronchus eingefüht, damit die anderseitige ganze Lunge von der Atmung ausgeschaltet wurde.

Bei der Durchsicht der Literatur über bisherige Untersuchungen, die sich von den obigen Gesichtspunkten aus experimentell mit der Analyse des Verhaltens des Eiweiss- und Kohlehydratspiegels in den Lungen befasst haben, habe ich, soweit die Literatur mir zugänglich ist, die Angaben von nur einigen Autoren gefunden. Mauriac u. Dumas ${ }^{2)}$ haben an Hunden nachgewiesen, dass wenn ein künstlicher Pneumothorax an der einen Iungen, denen normalerweise eine zuckerspaltende Funktion zukommt, angelegt ist, die betreffende Lunge gegenüber der intakten Lunge auf der gesunden Seite einen erhöhten Zuckergehalt aufweist; auf Grund dieser Beobachtung kamen genannten Autoren zum Schluss, dass die Lunge durch den Pneumothorax eine Abschwächung ihrer zuckerspaltenden Funktion erleidet. Od a ${ }^{3)}$ hat an Kaninchen festgestellt, dass die Lunge, die normalerweise den Blutzucker produziert und die Blutmilchsäure verbraucht, beim Anlegen des Pneumothorax das umgekehrte Verhalten, also den Zuckerverbrauch und die Milchsäureproduktion kundgibt. Des weiteren hat $\mathrm{Kasuga^{4 }}$ in Übereinstimmung mit Mauriac u. Dumas ${ }^{2}$ der Lunge eine zuckerspaltende Funktion zugesprochen und hinzugefügt, dass diese Funktion auch beim Anlegen des Pneumothorax ohne nachweisbare $A b-$ schwächung fast normal weiter erhalten bleibt. Mit Bezug auf die experimentelle Pneumonie hat Tomio $\mathrm{ka}^{5\}}$ Versuche an Kaninchen angestellt und ist der Meinung, dass die normale Lunge eine zuckerspaltende Funktion besitzt, bei experimentell erzeugter Pneumonie jedoch in ihrer Funktion herabgesetzt ist. $\mathrm{Ts} u \mathrm{ji}^{\mathrm{G}}{ }^{\mathrm{\gamma}}$ hat bei Kaninchen die experimentelle Pneumonie hervorgerufen und kam nach dem Tode des Versuchstiere durch die Analyse des autolytischen Verhaltens des Lungengewebes zum Ergebnis, dass bei der Pneumonie der

2) Ma u riac n. Duma s, C. r. Soc. Biol. Paris, 1924, 90, 1050.

3) Od a, Nippon Gekagakkai Zassi, 1937, 38, 689.

4) Ka s g ga, Kyoto Furitu Ikadajgaku Zassi, 1938, 23, 326.

5) Tomioka, Zikken Syokakibyogaku, 1928, 3, 1235.

6) Ts uji, Ibid., 1939, 14, 478 . 
Eiweissstơff wechsel in der Lunge erheblich gesteigert ist.

Nach obigen Angaben über das Verhalten der Kohlehydrate herrschen die Meinungsverschiedenheiten zwischen verschieden Autoren. Über das Verhalten des Eiweisses liegt nur ein Experiment von $\mathrm{Ts} u \mathrm{ji}^{\mathrm{b})}$ vor, er hat indessen Bestimmungen des Eiweisses nicht direkt an dem Blut, welches durch die Lungen passierte, ausgeführt, demgemäss die Funktionsänderungen der pathologischen Lungenzellen in bezug auf den Eiweisstoff wechsel überhaupt nicht geprüft.

Aus Rücksicht darauf wurden mit derselben Methode, wie sie in der ersten Mitteilung ${ }^{1)}$ geschildert wurde, vorliegende Versuche unter oben exwähnten Gesichtspunkten angestellt.

Versuchsmethode: Als Versuchstiere kamen gesunde Kaninchen von ca. $2 \mathrm{~kg}$ Körpergewicht zur Anwendung. Zwecks Anlegung des künstlichen Pneumothorax ist bei Kaninchen die atmosphärische Luft in Mengen von $15 \mathrm{ccm}$ pro $\mathrm{kg}$ Körpergewicht in die rechte Pleurahöhle zugeführt worden und nachdem Versuchstiere eine Std. lang ruhig liegen gelassen worden waren, wurde die Untersuchung durchgefiuhrt. Zwecks Erzeugung der experimentellen Pleuritis wurde 1 ccm einer 5 \%iger Ameisensäurelösung in die rechte Pleurahöhle des Kaninchens infundiert und nachdem das Exsudat sich in der Pleurahöhle maximal angesammelt hatte, also nach Ablauf von ca. 24 Std. nach Ameisensäurezufuhr wurde mit dem Versuch begonnen. Bei der Ausschaltung der einseitigen Lunge wurde so vorgegangen, dass man bei Kaninchen die Luftkanüle in den linken Hauptbronchus und zwar bis in den ein wenig unterwärts der Bifurkationsstelle gelegenen

Tabelle

Versuch beim künstiohen

\begin{tabular}{|c|c|c|c|c|c|c|c|c|c|}
\hline \multirow{3}{*}{ 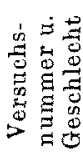 } & \multirow{3}{*}{ 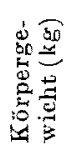 } & \multirow{3}{*}{ 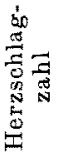 } & \multirow{3}{*}{ 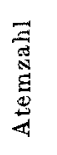 } & \multicolumn{3}{|c|}{ Hä moglobin } & \multicolumn{3}{|c|}{ Serumeiweiss } \\
\hline & & & & \multicolumn{2}{|c|}{$\mathbf{g} / \mathrm{d} \mathbf{l}$} & \multirow{2}{*}{$\frac{\mathrm{L}-\mathrm{R}}{\mathbf{R}} \times 100$} & \multicolumn{2}{|c|}{$\%$} & \multirow{2}{*}{$\frac{\mathrm{L}-\mathrm{R}}{\mathrm{R}} \times 100$} \\
\hline & & & & $\mathrm{R}$ & L & & $\mathbf{R}$ & $\mathrm{L}$ & \\
\hline 1 우 & 1,82 & 165 & 130 & 13,36 & 13,39 & $+0,2$ & 6,23 & 6,12 & $-1,8$ \\
\hline $2 \delta$ & 1,60 & 190 & 140 & 14,54 & 14,60 & $+0,4$ & 6,12 & 6,08 & $-0,6$ \\
\hline 36 & 1,92 & 192 & I 10 & 14,14 & 14,19 & $+0,3$ & 5,97 & 5,83 & $-2,3$ \\
\hline $4 \delta$ & 1,98 & 190 & 106 & 13,67 & 13,72 & $-0,4$ & 5,53 & 5,45 & $-1,4$ \\
\hline 59 & 1,60 & 185 & 128 & 14,31 & 14,31 & \pm 0 & 5,27 & 5,18 & $-1,7$ \\
\hline $6 \delta$ & 1,72 & 190 & 129 & 13,81 & 13,89 & $+0,6$ & 6,14 & 6,14 & \pm 0 \\
\hline 76 & 1,68 & 140 & 130 & 14,55 & 14,60 & $+0,3$ & 6,77 & 6,75 & $-0,3$ \\
\hline \multirow{2}{*}{\multicolumn{4}{|c|}{$\begin{array}{l}\text { Dursehnittswert } \\
\text { Durchschnitt der } \\
\text { normalen Zahlen }\end{array}$}} & 14,05 & 14,10 & $+0,3$ & 6,00 & 5,93 & $-1,2$ \\
\hline & & & & 14,20 & 14,27 & $+0,5$ & 6,08 & 5,98 & $-1,3$ \\
\hline
\end{tabular}

R: Blut ans der rechten Herzkammer. L: Blut aus der linken Herzkammer. 
Ort einfuhrte, um die rechte ganze Lunge von der Atemtätigkeit vollkommen auszuschalten, und die Untersuchung wurde eine Stunde danach durchgeführt. Die experimentelle Pneumonie wurde in der Weise provoziert, dass man eine $0,1 \%$ ige Silbernitratlösung in Mengen von $1,5 \mathrm{ccm}$ pro $\mathrm{kg}$ Körpergewicht in die Trachea infundierte. Der Versuch wurde nach ausgesprochener Entwicklung der Pneumonie an beiden Lungen, nämlich in 5-7 Tagen nach Einverleibung des Pharmacums angestellt. Nach Beendigung des Versuchs hat man sich durch Sektion von dem Bestehen der pneumonischen Entzündung an beiden Lungen überzengt. Die technischen Massnahmen der Blutentnahme und Bestimmungen waren dieselben, welche in der ersten Mitteilung') beschrieben wurden.

\section{Bestimmungen beim künstlichen}

$$
\text { Pneumothorax. }
$$

Daten, welche bei $7 \mathrm{Kaninchen} \mathrm{mit} \mathrm{dem} \mathrm{einseitigen} \mathrm{Pneumothorax}$ durch Bestimmungen an dem der Lunge zuströmenden und dem daraus abströmenden Blut gewonnen wurden, sind in Tab. 1 wiedergegeben. Der bequemen Vergleichbarkeit halber sind im unteren Teil der Tab. 1 die in der ersten Mitteilung ${ }^{1)}$ durch Bestimmungen an normalen Kaninchen gewonnenen Durchschnittswerte beigefügt angegeben.

Hämoglobin erfuhr nach der Lungenpassage des Blutes eine geringe Zunahme, die im Mittel $0,3 \%$ betrug und welche von derselben bei der normalen Lunge nicht wesentlich verschieden war.

1.

Pneumothorax auf der einen Seite.

\begin{tabular}{|c|c|c|c|c|c|c|c|c|c|c|c|}
\hline \multicolumn{3}{|c|}{ K. o. D. } & \multicolumn{3}{|c|}{ Druck pro $\%$} & \multicolumn{3}{|c|}{ Blutzucker } & \multicolumn{3}{|c|}{ Blutmilehsäure } \\
\hline \multirow{2}{*}{\multicolumn{2}{|c|}{$\frac{\mathrm{mm} \mathrm{H} \mathrm{H}_{2} \mathrm{O}}{\mathrm{R}: \mathrm{L}}$}} & \multirow{2}{*}{$\frac{L-R}{R} \times 100$} & \multicolumn{2}{|c|}{$\mathrm{mm} \mathrm{H}_{2} \mathrm{O}$} & \multirow{2}{*}{$\frac{\mathrm{L}-\mathrm{R}}{\mathrm{R}} \times 100$} & \multicolumn{2}{|c|}{$\mathrm{mg} / \mathrm{dl}$} & \multirow{2}{*}{$\frac{L-R}{R} \times 100$} & \multicolumn{2}{|c|}{$\mathrm{mg} / \mathrm{dl}$} & \multirow{2}{*}{$\frac{\mathrm{L}-\mathrm{R}}{\mathrm{R}} \times 100$} \\
\hline & & & $\mathrm{R}$ & $\mathrm{L}$ & & & $\mathrm{L}$ & & $\mathrm{R}$ & $\mathbf{L}$ & \\
\hline $\begin{array}{l}288 \\
267 \\
265 \\
248 \\
223 \\
274 \\
277\end{array}$ & $\begin{array}{l}282 \\
265 \\
256 \\
242 \\
217 \\
270 \\
270\end{array}$ & $\begin{array}{l}-2,1 \\
-0,7 \\
-3,4 \\
-2,4 \\
-2,7 \\
-1,4 \\
-2,5\end{array}$ & $\begin{array}{l}46,2 \\
43,6 \\
44,4 \\
44,8 \\
42,3 \\
44,6 \\
40,9\end{array}$ & $\begin{array}{l}46,1 \\
43,6 \\
43,9 \\
44,4 \\
41,9 \\
44,0 \\
40,0\end{array}$ & $\begin{array}{l}-0,2 \\
\pm 0 \\
-1,1 \\
-0,9 \\
-0,9 \\
-1,3 \\
-2,2\end{array}$ & $\begin{array}{l}119 \\
110 \\
132 \\
129 \\
138 \\
146 \\
131\end{array}$ & $\begin{array}{l}124 \\
115 \\
132 \\
127 \\
141 \\
146 \\
134\end{array}$ & $\begin{array}{l}+4,2 \\
+4,5 \\
\pm 0 \\
-1,5 \\
+2,2 \\
\pm 0 \\
+2,3\end{array}$ & $\begin{array}{l}30,21 \\
23,79 \\
36,00 \\
38,57 \\
40,50 \\
33,43 \\
22,50\end{array}$ & $\begin{array}{l}28,93 \\
22,50 \\
34,71 \\
39,86 \\
39,86 \\
34,07 \\
21,85\end{array}$ & $\begin{array}{r}-4,2 \\
-5,4 \\
-3,6 \\
+3,3 \\
-1,6 \\
+1,9 \\
-3,3\end{array}$ \\
\hline $\begin{array}{l}268 \\
263\end{array}$ & $\begin{array}{l}256 \\
256\end{array}$ & $\begin{array}{r}-2,7 \\
-2,3\end{array}$ & $\begin{array}{l}43,8 \\
43,0\end{array}$ & $\begin{array}{l}43,4 \\
42,7\end{array}$ & $\begin{array}{l}-0,9 \\
-0,7\end{array}$ & $\begin{array}{l}129 \\
100\end{array}$ & $\begin{array}{l}131 \\
103\end{array}$ & $\begin{array}{l}+1,5 \\
+3,0\end{array}$ & $\begin{array}{l}32,14 \\
31,07\end{array}$ & $\begin{array}{l}31,68 \\
29,61\end{array}$ & $\begin{array}{l}-2,0 \\
-4,7\end{array}$ \\
\hline
\end{tabular}


Serumeiweiss zeigte mit Ausnahme eines Versuchs, wo keine Schwankung anzutreffen war, in übrigen 6 Versuchen allenthalben mehr oder weniger Abnahme, welche im Mittel 1,2\% betrug.

K.o.D. sank nach der Lungenpassage des Blutes ohne Ausnahme ab. Dieses Absinken belief sich im Durchschnitt auf 2,7\%. K.o.D. für $1 \%$ Eiweiss (im folgenden kurz Druck pro \%) erniedrigte sich, ausgenommen einen Versuch mit unverändertem Wert, in allen übrigen Versuchen, diese Erniedrigung bezifferte sich auf durchschnittlich 0,9 $\%$.

Wenn man oben erwähnte Werte mit den Werten in derNorm vergleicht, so ergibt sich: Die Abnahme des Eiweisses weicht von der Norm nicht ab. K.o.D. und Druck pro \% erleiden anscheinend stärkere Erniedrigungen als in der Norm; diese Differenz ist jedoch so gering, dass man sie als in normalen Grenzen liegend ansehen darf. Es hat sich also herausgestellt, dass bei dem einseitig angelegten Pneumothorax die durch Lungenpassage zustande gekommene Veränderung des Bluteiweisses sowohl quantitativ wie auch qualitativ in nahezu gleichem Masse wie in der Norm erfolgt.

Was die Veränderungen des Kohlehydratspiegels anbetrifft, traten in 4 von 7 Versuchen (Versuch 1, 2, $5 u$. 7) die Blutzuckerzunahme und die Blutmilchsäureabnahme in annähernd gleichen Graden wie in der Norm auf. Bei übrigen 3 Versuchen liegen die Verhültnisse anders. Bei Versuch 3 trat nämlich bei der Stabilität des Zuckers die Abnahme der Milchsäure auf, es wurden ferner bei Versuch 4 dieZuckerabnahme und die Milchsäurezunahme, bei Versuch 6 beim Konstantbleiben des Zuckerspiegels die Milchsäurezunahme angetroffen. Aus oben erwähnten Ergebnissen geht also ohne weiteres hervor, dass beim einseitigen Pneumothorax, die Funktionsfähigkeit der Lungenzellen zur Zuckerbildung und Milchsäureelimination meistens ungestört erhalten bleibt, wobei aber zuweilen infolge von eventuellen Funktionsstörungen gleichsam ein Zustandsbild resultiert, wie es bei Inhalation von $\mathrm{O}_{2-}-$ armer Luft in der ersten Mitteilungil beobachtet wurde. Dies rührt meines Erachtens davon her, dass durch individuelle Verschiedenheit der Versuchstiere auch beim einseitigen Pneumothorax die $\mathrm{O}_{2}$-Versorgung der Lungenzellen erschwert wird. Dieses Ergebnis stimmt zum Teil mit Angaben von $\mathrm{Oda}^{3)}$ überein.

Von den spärlichen literarischen Angaben über die Veränderungen im kleinen Kreislauf, welche beim künstlichen Pneumothorax vorkommen, ist bereits im Eingang die Rede gewesen; fahnden wir nunmehr auf die Angaben der Vorgänger über die beim gleichen Eingriff im Blut 
des grossen Kreislaufs auftretenden Veränderungen des Eiweiss- und Kohlehydratspiegels, so finden wir bezüglich des Bluteiweisses folgende Arbeiten. Kimura u. Kato $0^{7)}$ und Mine ya ${ }^{8}$ haben bei Kaninchen nach der Anlegung des Pneumothorax im Blut die Zunahme des Rest$\mathrm{N}$ gefunden, während $\mathrm{Hashimoto} 0^{97}$ hingegen die Abrahme konstatierte. Über das Verhalten der Kohlehydrate hat Ric $\mathrm{ci}^{10)}$ bei Kranken mit der Lungentuberkulose nach der Anlegung des Pneumothorax die Hyperglykämie festgestellt; Yagishita u. Tatsumi ${ }^{11)}$ und Ikeu ti ${ }^{122}$ fanden unter gleichen Bedingungen eher eine Abnahme des Blutzuckers, Michelis u. Gic cher ${ }^{13}$ wollen die Zunahme der Blutmilchsäure nachgewiesen haben. Diesbezügliche Angaben lauten demnach sehr widersprechend. Bei Kranken gelingt es allerdings schwer, einheitliche Resultate zu erzielen, weil die Intensität des Krankheitsprozesses des betreffenden Individuums und der Grad des therapeutisch anzulegenden Pneumothorax je nach jeweiligen Umständen sich unterschiedlich gestalten.

Im Tierversuch haben Sasaki ${ }^{14)}$, Shim omu ra ${ }^{15)}$, Mine ya ${ }^{87}$ u.a. an Kaninchen, welche dem Pneumothorax unterworfen wurden, die Zunahme des Blutzuckers konstatiert und Domoricis), Hashimoto ${ }^{9)}$, Min e y ${ }^{8)}$ u.a. fanden die Zunahme der Blutmilchsäure. Demzufolge scheinen beim Pneumothorax im Blut des grossen Kreislaufes Vermehrungen des Blutzuckers sowie der Blutmilchsäure aufzutreten. Nach vorliegenden Versuchsergebnissen sind die Lungenzellen infolge des im Gefolge vom Pneumothorax vorkommenden $\mathrm{O}_{2}$-Mangels unter Umständen in ihrer Funktion, den Zucker zu bilden und die Milchsäure zu eliminieren, gestört, so dass nach dem Durchgang des Blutes durch die Lunge die Zuckerabnahme und die Milchsäurezunahme zustande kommen, in den meisten Fällen aber weist die Lungenfunktion keine Abweichung von der Norm auf.

\footnotetext{
7) Kimura u. Ka to, Kekkaku, 1931, 9, 567.

8) Mi neya, Nippon Naikagakkai Zassi, 1938, 25, 1561 u. 1577.

9) Hashimoto, Kyoto Furitu Ikadaigaku Zassi, 1935, 15, 145.

10) Ricci, Tuberculosi, 1929, 21, 1; ref. in Kongresszbl. f. ges. inn. Med., 1929, 54, 390.

11) Yagishita u. Tatsumi, Kyoto Furitsu Ikadaigakn Zassi, 1936, 18, 691 u. $1937,19,677$.

12) Ik euti, Kekkaku, 1937, 17, 519.

13) Michelis u. Gicehero, Arch. Sci. Med., 1938, 65, 245.

14) Sasakj, Zyuzenkai Zassi, 1932, 37, 1193.

15) Shimomura, Kyoto Igaku Zassi, 1937, 34, 344.

16) Domori, Zyuzenkai Zassi, 1935, 40, 1658.
} 
Tabelle

Versuch bei exsudativer

\begin{tabular}{|c|c|c|c|c|c|c|c|c|c|c|}
\hline \multirow{3}{*}{ 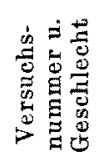 } & \multirow{3}{*}{ 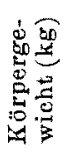 } & \multirow{3}{*}{ 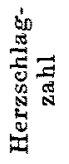 } & \multirow{3}{*}{ 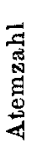 } & \multirow{3}{*}{ 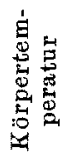 } & \multicolumn{3}{|c|}{ Hämoglobin } & \multicolumn{3}{|c|}{ Serumeiweiss } \\
\hline & & & & & \multicolumn{2}{|c|}{$\mathrm{g} / \mathrm{dl}$} & \multirow{2}{*}{$\frac{\mathrm{L}-\mathrm{R}}{\mathrm{R}} \times 100$} & \multicolumn{2}{|c|}{$\%$} & \multirow{2}{*}{$\frac{\mathrm{L}-\mathrm{R}}{\mathrm{R}} \times 100$} \\
\hline & & & & & $\mathbf{R}$ & $\mathbf{L}$ & & $\mathbf{R}$ & $\mathrm{L}$ & \\
\hline 1 令 & 2,10 & 236 & 192 & 38,2 & 13,94 & 14,00 & $+0,4$ & 5,72 & 5,51 & $-3,7$ \\
\hline 2 옹 & 1,90 & 186 & 158 & 39,5 & 14,24 & 14,35 & $+0,8$ & 5,94 & 5,75 & $-3,2$ \\
\hline $3 \hat{\delta}$ & 1,92 & 198 & 182 & 38,2 & 23,64 & 13,75 & $+0,8$ & 6,38 & 6,12 & $-4,1$ \\
\hline 4 우 & 2,25 & 216 & 163 & 38,8 & $\mathrm{I} 4,16$ & 14,27 & $+0,8$ & 5,70 & 5,53 & $-3,0$ \\
\hline 5 令 & 1,82 & 145 & 132 & 38,2 & 14,30 & 14,30 & \pm 0 & 6,12 & 6,12 & \pm 0 \\
\hline 6 우 & 1,90 & 192 & 148 & 37,8 & 13,97 & 14,24 & $+1,9$ & 6,34 & 6,27 & $-1,1$ \\
\hline 7 令 & 2,10 & 165 & 128 & 37,5 & 14,55 & 14,80 & $+1,7$ & 6,23 & 6,12 & $-1,8$ \\
\hline \multicolumn{5}{|c|}{ Dursehnittswert } & 14,15 & 14,24 & $+0,6$ & 6,06 & 5,92 & $-2,3$ \\
\hline
\end{tabular}

R: Blut aus der rechten Herzkammer. L: BIut aus der linken Herzkammer.

Die Milchsäurezunahme, welche oben genannte verschiedene Forscher beim Pneumothorax im Blut des grossen Kreislaufes nachgewiesen haben wollen, dürfte zum Teil auf die Milchsäurezunahme in den Lungen bezogen werden. Die von einzelnen Autoren beobachtete Zunahme des Blutzuckers lässt sich durchaus nicht mit der Beeinflussung des intermediären Kohlehydratstoffwechsels in der Lunge erklären, sondern sie dürfte höchstwahrscheinlich den im Gefolge vom Pneumothorax sekundär einsetzenden Veränderungen in anderen als der Lunge, dem intermediären Kohlehydratstoff wechsel teilnehmenden Organen zugeschrieben werden.

\section{Bestimmungen bei experimentell erzeugter Pleuritis.}

Versuchsresultate, welche an 7 Kaninchen, bei denen die rechtsseitige exsudative Pleuritis experimentell durch Ameisensäure erzeugt wurde, erhalten wurden, sind in Tab. 2 zusammengestellt.

Hämoglobin erfuhr, ausgenommen einen Versuch, wo der Hämoglobinwert konstant blieb, nach Durchgang des Blutes durch die Lunge in allen übrigen Versuchen eine Zunahme von durchschnittlich 0,6\%, die von der Norm kaum verschieden war.

Serumeiweiss erfuhr mit Ausnahme eines Versuchs mit konstantem Wert, in allen übrigen Versuchen nach der Lungenpassage des Blutes im Durchschnitt eine prozentische Abnahme von 2,3\%.

K. o. D. erniedrigte sich ohne Ausnahme, diese Frniedrigung betrug durchschnittlich 3,7\%. Diese Erniedrigung war deutlich grösser als in der Norm. 
2.

Pleuritis auf der einen Seite.

\begin{tabular}{|c|c|c|c|c|c|c|c|c|c|c|c|}
\hline \multicolumn{3}{|c|}{ K. o. D. } & \multicolumn{3}{|c|}{ Druck pro \% } & \multicolumn{3}{|c|}{ Blutzneker } & \multicolumn{3}{|c|}{ Blutmilehsäure } \\
\hline \multicolumn{2}{|c|}{ 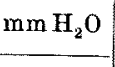 } & \multirow{2}{*}{$\frac{L-R}{R} \times 100$} & \multicolumn{2}{|c|}{$\mathrm{min} \mathrm{H}_{2} \mathrm{O}$} & \multirow{2}{*}{$\frac{\mathrm{L}-\mathrm{R}}{\mathrm{R}} \times 100$} & \multicolumn{2}{|c|}{$\mathrm{mg} / \mathrm{dl}$} & \multirow{2}{*}{$\frac{L-R}{R} \times 100$} & \multicolumn{2}{|c|}{$\mathrm{mg} / \mathrm{dl}$} & \multirow{2}{*}{$\frac{\mathbf{L}-\mathbf{R}}{\mathbf{R}} \times 100$} \\
\hline $\mathrm{R}$ & $\mathbf{L}$ & & $\mathbf{R}$ & $\mathbf{L}$ & & $\mathrm{R}$ & $\mathrm{L}$ & & $\mathbf{R}$ & $\mathrm{L}$ & \\
\hline 231 & 220 & $-4,8$ & 40,4 & 40,2 & $-0,5$ & 141 & 134 & $-5,0$ & 41,14 & 43,71 & $+6,2$ \\
\hline 235 & 226 & $-3,8$ & 39,6 & 39,3 & $-0,7$ & 131 & 129 & $-1,5$ & 38,57 & 39,86 & $+3,3$ \\
\hline 244 & 234 & $-4,1$ & 38,2 & 38,2 & \pm 0 & 125 & 125 & \pm 0 & 39,21 & 39,86 & $+1,6$ \\
\hline 240 & 232 & $-8,3$ & 42,1 & 41,9 & $-0,5$ & 141 & 139 & $-1,4$ & 45,00 & 46,29 & $+2,9$ \\
\hline 243 & 240 & $-1,6$ & 39,7 & 39,2 & $-1,2$ & 136 & 136 & \pm 0 & 47,57 & 46,93 & $-1,3$ \\
\hline 244 & 235 & $-3,7$ & 38,5 & 37,5 & $-2,6$ & 127 & 124 & $-2,4$ & 44,39 & 45,00 & $+1,4$ \\
\hline 259 & 247 & $-4,6$ & 41,6 & 40,3 & $-1,3$ & 150 & 150 & \pm 0 & 41,79 & 39,86 & $-4,6$ \\
\hline 242 & 233 & $-3,7$ & 40,0 & 39,5 & $-1,2$ & 136 & 134 & $-1,5$ & 42,52 & 43,07 & $+3,6$ \\
\hline
\end{tabular}

Druck pro \% nahm, mit Ausnahme eines Versuchs mit konstantem Wert, nach der Lungenpassage des Blutes in übrigen 6 Versuchen mehr oder weniger ab; die prozentische Abnahme betrug im Durchschnitt 1,2 $\%$. Diese Zahl war gegenüber der Norm gewissermassen vergrössert.

Blutzucker erfuhr nach der Lungenpassage in 4 von 7 Versuchen, im Gegensatz zur Norm, eine prozentische Abnahme von 1,4-5,0\%, während er in übrigen 3 Versuchen keine Schwankung aufwies. Blutmilchsäure erfuhr nach der Lungen passage in 2 Versuchen Abnahme, in übrigen 5 Ver'suchen im Gegensatz zur Norm Zunahme, die 1,4-6,2 $\%$ betrug.

Wenn man das Verhalten der oben angeführten Grössen den einzelnen Werten nach näher betrachtet, so ergibt sich: Es waren 4 Versuche (Versuch 1, 2, 4 u. 6), in denen, im Gegensatz zur Norm, durch Blutpassage durch die Lunge die Zuckerabnahme und die Milchsäurezunahme auftraten, unter übrigen 3 Versuchen befand sich Versuch 3 , in welchem beim Konstantbleiben des Zuckerspiegels die Zunahme der Milchsäure angetroffen wurde und in Versuch 5 u. 7 wurde beim unveränderten Zuckerwert die Milchsäure vermindert gefunden. Kurzum, unter obigen 7 Versuchen befanden sich 2 Versuche (Versuch $5 \mathrm{u}$. 7), bei denen sich die Kohlehydratveränderung, wenn auch beim Ausbleiben der Zuckerzunahme, doch aus der Milchsäureabnahme schliessend, in annähernd analoger Weise wie in der Norm verhielt, in übrigen 4 Versuchen (Versuch 1, 2, 4 u. 6) wurde das der Nor'm entgegengesetzte Verhalten konstatiert; ein noch restierter Versuch gilt als ein Übergangstyp zwischen den beiden Formen. 
Aus alledem geht klar hervor, dass, wenn man experimentell einseitige exsudative Pleuritis provoziert, nach Durchgang des Blutes durch die Lunge die Abnahme des Bluteiweisses und dessen k.o.D. deutlich grösser als in der Norm ist, wobei auch die Erniedrigung des Drucks pro \% gewissermassen stärker ist. Es treten nämlich auch unter normalen Verhältnissen nach der Lungenpassage die Abnahme des Bluteiweisses und die Verschiebung der Eiweissteilchen nach grober Seite hin in Erscheinung, bei der Pleuritis erfolgt die Veränderung in gleicher Richtung, deren Grad aber gewissermassen stärker wie in der Norm ist.

Während beim künstlichen Pneumothorax im vorherigen Kapitel die Veränderungen von Bluteiweiss und dessen k.o.D. fast keine Unter* schiede von der Norm zeigten, treten einschlägige Veränderungen bei exsudativer Pleuritis in etwas stärkerem Masse wie in der Norm in Erscheinung. Der Grund hierfür dürfte wohl darin gelegen sein, dass das die Pleuritis verursachende Moment, also der entzündliche Reiz der. Ameisensäure sich bis auf den der Pleura pulmonalis nahegelegenen Lungenteil übergreift, wo neben gesteigerter Eiweisszersetzung sich die Permeabilität der hier befindlichen Lungenkapillaren steigert, und wo kleine Eiweissteilchen, welche die Kapillarwand unter normalen Verhältnissen keineswegs durchlässt, teilweise von hier heraus in das Lungengewebe oder das Exsudat übergehen. Dass bei der Pleurits im Exsudat erscheinendes Eiweiss von dem Bluteiweiss herstammt, kann ohne weiteres gefolgert werden aus Versuchsresultaten von Govaerts ${ }^{17}$, Schade u. a. ${ }^{18}$, Iversen u. Nakazawa ${ }^{19}$, Iversen u. Johan$\mathrm{sen}^{20)}$, Kylin ${ }^{21)}$, Meyer u. Friedheim ${ }^{22)}$, Åmann ${ }^{23)}$ und Puddu ${ }^{24)}$, die alle das Bluteiweiss und Exsudateiweiss sowie k.o.D.-Werte in beiden Eiweissarten vergleichend bestimmt haben. An hiesiger Klinik machte Nishiyama ${ }^{25}$ früher die Beobachtung, dass, obwohl bei der Pleuritis das Bluteiweiss und dessen k.o.D. mit dem Krankheitsverlauf erhebliche Schwankungen aufweisen, in Stadium incrementi jedoch Werte der oben genannten Grössen im allgemeinen herabgesetzt und

17) Govaerts, C. r. Soc. Biol. Paris, 1924, 91, 116.

18) Schade, Claussen, Häbler, Hoff, Mochizuki u. Birner, Zeitschr. f. ges. exp. Med., 1926, 49, 334.

19) I versen u. Nakazawa, Biochem. Zeitschr., 1927, 191, 307.

20) I versen u. Johansen, Klin. Wochenschr., 1929, 309.

21) Kylin, Acta med. Scandinay., 1929, 72, 561.

22) Meyer u. Fried hei m, Zeitschr. f. klin. Med., 1931, 119, 236.

23) Arm a n n, Zeitschr. f. ges. exp. Med, 1932, 85, 853.

24) Pud d u, Ibid., 1935, 95, 754.

25) Nishiyama, Tohoku Journ. Exp. Med., 1934, 22, 505. 
mit dem Eintritt der Heilung allmählich erhöht sind. Diese Beobachtung deckt sich mit dem volliegenden Resultat, dass bei der Pleuritis im Höhepunkt des Krankheitsprozesses im Blut, welches durch die Lunge passiert hat, die Verminderung des Eiweisses und die Erniedrigung des k.o.D. in stärkerem Masse als in der Norm zutage treten.

Alles in allem kann hervorgehoben werden, dass die Veränderung der Kohlehydrate hier im wesentlichen zwar gleiche Richtung, aber in stärkerem Grade wie beim künstlichen Pneumothorax im vorherigen Kapitel auftritt. Beim küinstlichen Pneumothorax geht die Funktion der Lungenzellen zur Zuckerbildung und zur Milchsäureelimination meistens ungestört vonstatten, wobei jedoch wegen der sich eventuell ereignenden Funktionsstörungen die Verminderung des Blutzuckers und die Vermehrung der Blutmilchsäure zustande kommen können. Bei exsudativer Pleuritis bingegen treten, ausgenommen nur wenige Versuche, wo, wie oben angefuhrt, eine wenn auch unvollkommen, doch derselben in der Norm angenährte Veränderung vorkommt, in der Mehrzahl der Fälle die Zuckerverminderung und die Milchsäurevermehrung in Erscheinung; es ist dies offenbar ein Hinweis darauf, dass die Funktion der Lungenzellen hier in weitgehend stärkerem Masse als beim künstlichen Pneumothorax geschädigt ist. Beim künstlichen Pneumothorax kann nämlich die Zirkulation in der kompensierten Lunge bis zu einem gewissen Grad bzw. annähernd normal vor sich gehen und die Zellfunktion des betreffenden Lungengewebes ziemlich intakt bleiben. Bei derjeniger Pleuritis, bei welcher das Exsudat sich im maximalen Grad angesammelt hat, ist die Elastizität der Lunge derselben Seite in stärkerem Ausmasse als bei dem Pneumothorax beeinträchtigt, wobei die Störung der Zirkulation in der betreffenden Lunge immer erheblicher sich geltend macht, und dadurch wird die zur normalen Funktionsleistung der Lungenzellen erforderliche Sauerstoffversorgung derselben von Seiten des Blutes ausgeprägter als beim Pneumothorax erschwert. Als eine noch andere denkbare Möglichkeit dürfte herangezogen werden, dass weil entzündliche Vorgänge durch Ameisensäure sich, wie oben bereits erörtert, bis auf einzelne Abschnitte der Lunge fortpflanzen, hier nebst gesteigerter Zuckerspaltung die Bildung. der Milchsäure einsetzen kann.

Im übrigen gibt es Autoren, welche bei Pleuritiden am Krankenbett die Bestimmungen von Blutzucker und Blutmilchsäure ausführten; die einen davon (Schelle ${ }^{26)}$, Oshima $a^{27)}$, Momose $e^{(3)}$ ) berichteten über die Stabilität bei-

26) Seheller, Münch. med. Wochenschr., 1926, 1879.

27) Oshima, Kekkaku, 1927, 5, 1179.

28) Momose, Ijisimbun, 1927, 697 . 
Tabelle

Versuch bei der Ausschaltung der

\begin{tabular}{|c|c|c|c|c|c|c|c|c|c|}
\hline \multirow{3}{*}{ 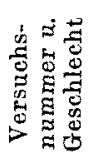 } & \multirow{3}{*}{ 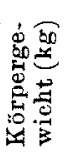 } & \multirow{3}{*}{ 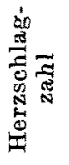 } & \multirow{3}{*}{ 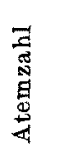 } & \multicolumn{3}{|c|}{ Hämoglobin } & \multicolumn{3}{|c|}{ Serumeiweiss } \\
\hline & & & & \multicolumn{2}{|c|}{$g / d l$} & \multirow{2}{*}{$\frac{L-R}{R} \times 100$} & \multicolumn{2}{|c|}{$\%$} & \multirow{2}{*}{$\frac{\mathbf{L}-\mathbf{R}}{\mathbf{R}} \times 100$} \\
\hline & & & & $\mathrm{R}$ & $\mathrm{L}$ & & $\mathrm{R}$ & $\mathrm{L}$ & \\
\hline I $\hat{o}$ & 1,85 & 232 & 120 & 14,30 & 14,43 & $+0,9$ & 6,36 & 6,23 & $-2,0$ \\
\hline 2 우 & 2,00 & 223 & 118 & 15,10 & 15,24 & $+0,9$ & 6,27 & 6,19 & $-1,3$ \\
\hline $3 \hat{8}$ & 1,80 & 192 & 132 & 13,83 & 14,05 & $+1,6$ & 6,75 & 6,51 & $-3,5$ \\
\hline 4 ช & 1,90 & 268 & 136 & 14,24 . & 14,32 & $+0,6$ & 5,49 & 5,43 & $-1,1$ \\
\hline 58 & 1,75 & 218 & 96 & 14,63 & 14,68 & $+0,3$ & 5,68 & 5,65 & $-0,5$ \\
\hline \multicolumn{4}{|c|}{ Durchschnittswert } & 14,42 & 14,55 & $+0,9$ & 6,11 & 6,00 & $-1,7$ \\
\hline
\end{tabular}

R: Blut aus der rechten Herzkammer. L: Blut aus der linken Herzkammer.

der Werte, während die anderen (Hatta u. Y $\mathrm{Yno} \mathrm{o}^{29)}$, T a tikaw ${ }^{20)}$ ) mehr oder weniger Zunahmen angaben. Wenn man indessen nur die Beeinflussung der Kohlehydrate durch Lungenpassage des Blutes allein ins Auge fasst, so wird man zum mindesten zu einer Zeit, wo die Exsudatansammlung das Maximum erreicht hat, in den meisten Fällen Blutzuckerabnahme und Milchsäurezunahme annehmen müssen.

\section{Bestimmungen bei Ausschaltung derganzen Lunge auf der einen Seite.}

Versuchsresultate, welche an 5 Kaninchen, bei denen die rechte Lunge durch das Einlegen der Luftkanüle in den linken Hauptbronchus von der Atmung vollkommen ausgeschalt wurde, durch Bestimmungen erhalten wurden, sind in Tab. 3 wiedergegeben.

Hämoglobin nahm nach Durchgang des Blutes durch die Lunge überall an Konzentration zu, die durchschnittliche prozentische Zunahme war gegenüber der Norm ziemlich erhöht, die Eindickung des Blutes war also nach der Lungenpassage etwas höhergradig als in der Norm.

Serumeiweiss, k.o.D. und Druck pro \% erfuhren nach der Lungenpassage des Blutes ohne Ausnahme Abnahmen; durchschnittliche prozentische Abnahme betrug der Reihe nach $1,7(0,5-3,5) \%, 3,0(1,4-6,3)$ $\%$ und $1,6(0,2-3,0) \%$. Diese Zifferzahlen waren im Vergleich mit entsprechenden Werten bei der normalen Lunge im allgemeinen gewissermassen vergrössert. Es stellt sich nämlich heraus, dass wenn

29) Hatta u. Yano, Zyuzenkai Zassi, 1928, 33, 2015.

30) Tatikawa, Mansyu Igaku Zassi, 1930, 12, 661. 
3.

einseitigen Lungenatmung.

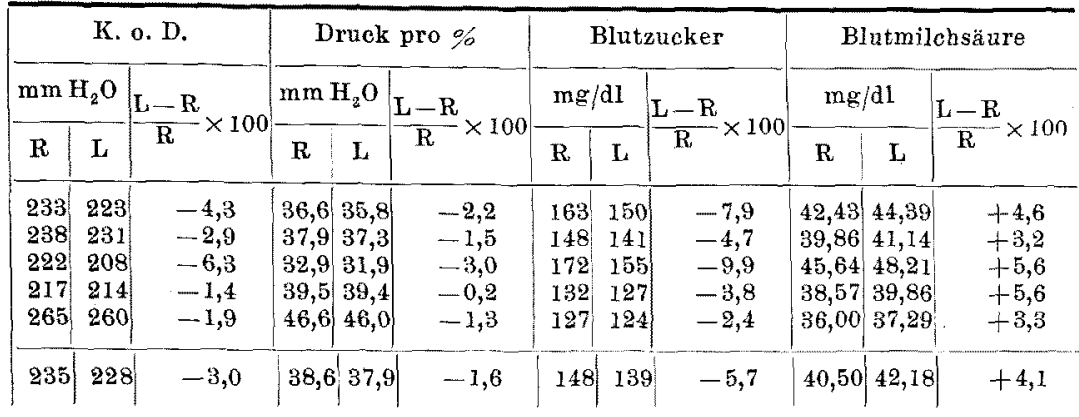

die eine Lunge vollkommen von der Atmung ausgeschaltet ist, das Bluteiweiss nach Durchgang des Blutes durch den kleinen Kreislauf in quantitativer und qualitativer Hinsicht etwas stärkere Veränderungen erleidet als bei der Funktionstüchtigkeit der beiden Lungen.

Im kleinen Kreislauf verhielt sich der intermediäre Kohlehydratstoffwechsel in sämtlichen Versuchen gerade umgekehrt wie die bei der normalen Lunge, indem der Blutzucker Verminderung, die Blutmilchsäure Vermehrung erfuhr; im Durchschnitt betrug die erstere $-5,7(2,4-9,9) \%$, die letztere $+4,1(3,2-5,6) \%$.

Da bei oben genannten Versuchen die Blutzirkulation in den beiden Lungen durch das Einlegen der Kanüle nicht wesentlich beeinflusst wurde, so ist es durchaus nicht angängig, oben geschilderte Veränderungen auf die Zirkulationsstörung zurückzuführen. Zudem befand sich die linke Lunge, die von der Atmung nicht ausgeschaltet wurde, natürlich im vollauf normalen Zustand. Bei der rechten Lunge liegen aber die Verhältnisse anders; weil hier beim hinlänglichen $\mathrm{O}_{2}$ Angebot von der Kreislaufseite doch derselbe von den Alveolen, also von der Luftseite völlig ausbleibt, dürften sich dadurch genannte Störungen einstellen können. Die gegenüber der Norm vergrösserte Zunahme des Hämoglobins dürfte wohl dahin gedeutet werden, dass, weil in der Lunge, worin kein Gaswechsel mehr stattfindet, die Permeabilität der Kapillaren naturgemäss gesteigert ist, ein Teil von dem Blutwasser mehr als sonst extravasal abgewandelt ist. ImZusammenhang damit ist auch anzunehmen, dass hierbei auch die Bluteiweisskörper, insbesondere relativ klein molekuläre Eiweissteilchen, also Eiweissfraktion der Albuminklasse zum Teil samt dem Blutwasser extra- 
vasal in das Lungengewebe übertreten, was zwangsläufig zur quantitativer Eiweissabnahme und qualitativ zur Verschiebung der Eiweissteilchen nach grobdisperser Seite hin führen muss.

Der Unstand, dass das Verhalten des Blutzuckers und der Blutmilchsäure gemeinsam gerade ins Gegenteil von demselben in der Norm umschlägt, lässt sich höchstwahrscheinlich dahin erklären, dass ausgeschaltete Lungenzellen infolge des Ausbleibens des $\mathrm{O}_{2}$-Angebotes von der Luftseite nicht mehr imstande sind, ihre funktionelle Tätigkeit, den Zucker zu bilden und die Milchsäure zu eliminieren, tüchtig zu leisten, so dass nunmehr in der betreffenden Lunge nur der Zuckerverbrauch und die Milchsëureproduktion sich geltend machen. Aus dem Vergleich der $\mathrm{O}_{2}$-Versorgung der Lungenzellen von Blutseite und Luftseite ergibt sich ohne weiteres, dass die $\mathrm{O}_{2}$-Versorgung von Seiten des Blutes derselben von der Luftseite nach steht. Dieses Ergebnis steht in annäherndem Einklang mit Befunden von $\mathrm{Oda} \mathrm{a}^{3}$, der nach Ausschaltung der einen Lunge, indem er einen aus Blei hergestellten Ball in der Hauptbronchus auf der einen Seite einführte, diesbezügliche Untersuchung ausführte.

4. Bestimmungen bei experimenteller Pneumonie.

Bei 7 Kaninchen wurde durch Zufuhr des Silbernitrates in die beiden Lungen experimentell die Pneumonie erzeugt, und im Stadium, wo der Krankheitsprozess seinen Gipfelpunkt erreichte, wurde Versuch angestellt (Tab. 4).

Tabelle

Tersuch bei experimentell erzeugter

\begin{tabular}{|c|c|c|c|c|c|c|c|c|c|c|}
\hline \multirow{3}{*}{ 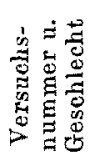 } & \multirow{3}{*}{ 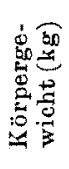 } & \multirow{3}{*}{ 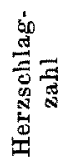 } & \multirow{3}{*}{ 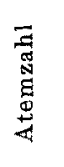 } & \multirow{3}{*}{ 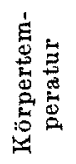 } & \multicolumn{3}{|c|}{ Hämoglobin } & \multicolumn{3}{|c|}{ Serumeiweiss } \\
\hline & & & & & \multicolumn{2}{|c|}{$\mathrm{g} / \mathrm{dl}$} & \multirow{2}{*}{$\frac{L-R}{R} \times 100$} & \multicolumn{2}{|c|}{$\%$} & \multirow{2}{*}{$\frac{\mathrm{L}-\mathrm{R}}{\mathrm{R}} \times 100$} \\
\hline & & & & & $\mathbf{R}$ & $L$ & & $\mathbf{R}$ & $\mathbf{L}$ & \\
\hline $1 \%$ & 1,82 & 225 & 158 & 40,5 & 8,97 & 9,18 & $+2,3$ & 5,53 & 5,34 & $-3,4$ \\
\hline 20 & 2,15 & 182 & 142 & 39,4 & 11,08 & 11,19 & + & 5,92 & 5,72 & $-3,4$ \\
\hline 3 3 & 2,60 & 168 & 120 & 38,9 & 12,27 & 12,38 & $+0,9$ & 5,47 & 5,27 & $-5,5$ \\
\hline 48 & 2,48 & 170 & 160 & 38,6 & 13,14 & 13,30 & $\begin{array}{r}1,2 \\
+1,2\end{array}$ & 6,14 & 5,92 & $-3,6$ \\
\hline 5 合 & 2,00 & 192 & 136 & 39,8 & 13,11 & 13,36 & $\begin{array}{r}1,0 \\
+1,9\end{array}$ & 5,68 & 5,43 & $-4,4$ \\
\hline 68 & 2,08 & 210 & 132 & 39,9 & 12,10 & 12,18 & $+0,7$ & 6,01 & 5,78 & $-3,8$ \\
\hline 7 우 & 1,89 & 198 & 118 & 38,8 & 12,35 & 12,49 & $+1,1$ & 5,97 & 5,77 & $-3,3$ \\
\hline \multicolumn{5}{|c|}{ Durschnittswert } & 11,86 & 12,01 & $+1,3$ & 5,82 & 5,60 & $-3,8$ \\
\hline
\end{tabular}

R: Blut aus der rechten Herzkammer.

L: Blut aus der linken Herzkammer. 
Hämoglobin erfuhr nach Durchgang des Blutes durch die Lunge gewaltige Zunahme, welche sich natürlich weitgehend grösser als in der Norm und auch ausgeprägter als bei dem Fall im vorherigen Kapitel, wo die eine Lunge von der Atmung ausgeschaltet wurde, erwies. Dies deutet zweifelsohne darauf hin, dass das Blut durch Durchgang durch die entzündeten Lungen bedeutend an Wasser verliert.

Serumeiweiss nahm ohne Ausnahme beträchtlich ab, die prozentische Abnahme betrug im Durchschnitt 3,8 (3,3-5,5) \%. K.o.D. erlitt auch erhebliche Erniedrigung, deren Prozentsatz im Mittel zu 8,4(5,8$12,0) \%$ ermittelt wurde. Da die Erniedrigung des k.o.D. höhergradig. als die Eiweissabnahme war, erfuhr auch Druck pro \% erhebliches Absinken, dessen Prozentsatz sich auf durchschnittlich 4,9 (2,2-9,1)\% belief. Nach alledem kann also gesagt werden, dass durch Durchgang des Blutes durch die entzündeten Lungen das Bluteiweiss quantitativ stärkere Abnahme als bei anderen Fällen erfährt, während sich zugleich qualitativ die Eiweissfraktionen erheblich nach grobdisperser Seite hin verschieben.

Was die Veränderungen von Blutzucker und Blutmilchsäure anbetrifft, kann man kurzgefasst so sagen, dass sie in weit stärkerem Masse als bei dem Fall, wo die eine Lunge von der Atmung ausgeschaltet wurde, auftraten. Bei sämtlichen 7 Versuchen traten nämlich ohne Ausnahme eine Abnahme des Blutzuckers und eine Zunahme der Blutmilchsäure in Erscheinung, wobei die Abnahme des Zuckers auf 9,3 $(6,9-10,8) \%$, die Zunahme der Milchsäure auf 5,4 $(4,1-8,2) \%$ bezifferte.

4.

Penumonie an beiden Lungen.

\begin{tabular}{|c|c|c|c|c|c|c|c|c|c|c|c|}
\hline \multicolumn{3}{|c|}{ K. o. D. } & \multicolumn{3}{|c|}{ Druek pro $\%$} & \multicolumn{3}{|c|}{ Blutzueker } & \multicolumn{3}{|c|}{ Blutmilchsãure } \\
\hline \multicolumn{2}{|c|}{$\mathrm{mm} \mathrm{H}_{2} \mathrm{O}$} & \multirow{2}{*}{$\frac{\mathrm{L}-\mathrm{R}}{\mathrm{R}} \times 100$} & \multicolumn{2}{|c|}{$\mathrm{mm} \mathrm{H} \mathrm{H}_{2} \mathrm{O}$} & \multirow{2}{*}{$\frac{\mathrm{L}-\mathrm{R}}{\mathrm{R}}-\times 100$} & \multicolumn{2}{|c|}{$\mathrm{mg} / \mathrm{dl}$} & \multirow{2}{*}{$-\frac{\mathrm{L}-\mathrm{R}}{\mathrm{R}} \times 100$} & \multicolumn{2}{|c|}{$\mathrm{mg} / \mathrm{dl}$} & \multirow{2}{*}{$-\frac{\mathrm{L}-\mathrm{R}}{\mathrm{R}} \times 100$} \\
\hline$R$ & Is & & $\mathbf{R}$ & $\mathrm{L}$ & & $\mathbf{R}$ & $\mathbf{L}$ & & $\mathbf{R}$ & $\mathbf{L}$ & \\
\hline $\begin{array}{l}183 \\
214 \\
173 \\
239 \\
228 \\
243 \\
215\end{array}$ & $\begin{array}{l}161 \\
198 \\
163 \\
222 \\
208 \\
218 \\
198\end{array}$ & $\begin{array}{r}-12,0 \\
-\quad 7,5 \\
-\quad 5,8 \\
-\quad 7,1 \\
-\quad 8,8 \\
-10,3 \\
-\quad 7,9\end{array}$ & $\begin{array}{l}33,1 \\
36,1 \\
31,6 \\
38,9 \\
40,1 \\
40,4 \\
36,0\end{array}$ & $\begin{array}{l}30,1 \\
34,6 \\
30,9 \\
37,5 \\
38,3 \\
37,7 \\
34,3\end{array}$ & $\begin{array}{l}-9,1 \\
-4,1 \\
-2,2 \\
-3,6 \\
-4,5 \\
-6,7 \\
-4,7\end{array}$ & $\begin{array}{l}131 \\
141 \\
131 \\
129 \\
152 \\
148 \\
146\end{array}$ & $\begin{array}{l}122 \\
131 \\
119 \\
115 \\
136 \\
136 \\
131\end{array}$ & $\begin{array}{r}-6,9 \\
-\quad 7,1 \\
-\quad 9,2 \\
-10,8 \\
-10,5 \\
-\quad 8,1 \\
-10,3\end{array}$ & $\begin{array}{l}37,93 \\
30,86 \\
39,21 \\
41,79 \\
43,07 \\
39,21 \\
38,57\end{array}$ & $\begin{array}{l}39,86 \\
32,14 \\
41,14 \\
43,71 \\
45,61 \\
42,33 \\
40,50\end{array}$ & $\begin{array}{l}+5,1 \\
+4,1 \\
+4,9 \\
+4,6 \\
+6,0 \\
+8,2 \\
+5,0\end{array}$ \\
\hline 213 & 195 & $-8,4$ & 36, & 348 & $-4,9$ & 140 & 127 & $-9,3$ & 38,66 & 40,76 & \\
\hline
\end{tabular}


Allerdings wurden Versuche an solchen Kaninchen angestellt, bei denen der Krankheitsprozess der Pneumonie sich intensiv abspielte, indem in den beiden Lungen uberall, vor allem aber im Unterlappen nachgewiesenermassen pneumonische Herde dicht nebeneinander verbreitet waren. Bei derartigen Tieren muss natürlich die Atemfläche der Lunge erheblich eingeschränkt sein, auch die Lungenzellen selbst sind durch die Entzündung stark geschädigt, wobei aber die Lungenzirkulation übernormal vor sich geht, weil die Gefässe der pneumonischen Lungen durch entzündliche Hyperämie erweitert sein müssen. Aus dieser Überlegung können für.die Beeinträchtigung der Funktionsleistung der Lungenzellen folgende Faktoren in Betracht gezogen werden: Erstens die gestörte $\mathrm{O}_{2}-$ Versorgung von der Luftseite infolge der verminderten Atemfläche, zweitens entzündliche Veränderungen der Lungenzellen selbst, ferner gesteigerte Permeabilität der Lungenkapillaren durch entzündliche Prozesse. Die beträchtliche Zunahme der Hämoglobinkonzentration lässt sich wohl durch den extravasalen Austritt des Blutwassers durch entzündliche Hyperämie und gesteigerte Permeabilität der Lungenkapillaren erklären. Die Eiweissabnahme und erhebliche Erniedrigungen von k.o.D. sowie Druck pro $\%$ dürften als dadurch entstanden aufgefasst werden, dass kleine Eiweissteilchen, für welche die Kapillarwand unter normalen Verhältnissen impermeabel ist, zusammen mit dem Blutwasser extravasel austreten und ferner dass weil die Lungenzellen sich im entzündeten Zustand befinden, wie $\mathrm{Tsu} \mathrm{j}^{\mathrm{i}^{6}}$ hervorgehoben hat, die Eiweisszersetzung in der Lunge in verstärktem Masse vor sich geht.

Fur Verminderung des Blutzuckers und Vermehrung der Blutmilchsäure werden zum mindesten zwei Faktoren in Erwägung gezogen. Erstens wegen der Erkrankung der Lungenzellen sind diese, wie bereits ofters angeführt, nicht mehr imstande, ihre normale funktionelle Leistungen, also die Zuckerbildung und Milchsäureeliminierung zu bewerkstelligen, zweitens infolge der pneumonischen Infiltration der Alveolen wird die $\mathrm{O}_{2}-$ Versorgung der erkrankten Lungenzellen von der Luftseite überaus erschwert. Dadurch erleidet das Blut nach Durchgang durch die entzündeten Lungen Erniedrigung am Zuckerspiegel und Zunahme am Milchsäuregehalte und zwar in erheblichem Ausmasse.

$$
\text { Zusammenfassung. }
$$

Behufs Einschränkung der Lungenzirkulation und der Atemfläche sowie zwecks Schädigung der Lungenzellen wurde bei Kaninchen auf 
der einen Seite der künstliche Pneumothorax angelegt oder die eine Lunge wurde von der Atmung ausgeschaltet, indem eine Luftkanüle in den Bronchus der anderseitigen Lunge eingelegt wurde. Es wurde - weiterhin die einseitige Pleuritis oder die beiderseitige Pneumonie experimentell erzeugt. Unter oben geschilderten verschiedenen pathologischen Verhältnissen wurden vor und nach dem Durchgang des Blutes durch die Lunge die Bestimmungen von Hämoglobin, Serumeiweiss, kolloid-osmotischem Druck (k.o.D.), Blutzucker und Blutmilchsäure vorgenommen. Auf obige Weise sind die Verhältnisse des intermediären Wasser-, Eiweiss- und Kohlehydratstoffwechsels der Lungenzellen unter pathologischen Zuständen untersucht worden.

1. Einseitiger Pneumothorax: Nach Durchgang des Blutes durch die Lunge nimmt die Hämoglobinkonzentration zu, während das Eiweiss und der k.o.D. abnehmen, die Grade dieser Veränderungen zeigen aber keine Unterschiede von denselben bei der normalen Lunge. Bezüglich des Blutzuckers und der Blutmilchsäure lässt sich sagen, dass es hier ebenso wie in der Norm meistens zur Zunahme des Zuckers und zur Abnahme der Milchsäure kommt, wobei aber mitunter eine Abnahme des Zuckers und eine Zunahme der Milchsäure auftreten können.

2. Experimentell erzengte exsudative Pleuritis auf der einen Seite: Hämoglobinkonzentration erfährt nach der Lungenpassage des Blutes eine Zunahme, die aber gegenüber der normalen Lunge keinen bemerkbaren Unterschied zeigt. Serumeiweiss, k.o.D. und Druck pro $\%$ nehmen nach der Lungenpassage gemeinschaftlich in gewissermassen grösserem Ausmass ab als bei der normalen Lunge.

Bezüglich des Blutzuckers und der Blutmilchsäure verhalten sie meistens in umgekehrtem Verhältnisse zur Norm, indem nämlich nach der Lungenpassage des Blutes der Zucker ab-, und die Milchsäure zunimmt, es sei denn, dass die letztere mitunter abnimmt.

3. Ausschaltung der einen Lunge von der Atmung: Nach Durchgang des Blutes durch die Lunge nimmt die Hämoglobinkonzentration zu, während der Serumeiweissgehalt, k.o.D. und Druck pro \% abnehmen, diese Veränderungen spielen sich in höherem Grade ab als in der Norm. Der Blutzucker weist Verminderung, die Blutmilchsäure Vermehrung auf.

4. Experimentell erzeugte Pneumonie an beiden Lungen: Nach der Lungenpassage erfährt die Hämoglobinkonzentration eine Zunahme, das Serumeiweiss, k.o.D. und Druck pro \% erleiden gleichfalls Erniedrigungen, der Blutzucker vermindert sich, die Blutmilchsäure ver- 
mehrt sich. Die Zu- und Abnahmen betreffender Grössen erfolgen also in stärkerem Ausmasse als bei der Ausschaltung der einen Lungen von der Atmung.

Die Deutungen aller oben erwähnten Veränderungen sind an betreffenden Stellen auseinandergesetzt. 